W. ZHANG, Z. ZHANG, J.-C. TANG, J.-T. CHE, H.-Y. ZHANG, J.-H. CHEN*,Z. YANG* (PEKING UNIVERSITY AND PEKING UNIVERSITY SHENZHEN GRADUATE SCHOOL, P. R. OF CHINA)

Total Synthesis of (+)-Haperforin G

J. Am. Chem. Soc. 2020, 142, 19487-19492, DOI: 10.1021/jacs.0c10122.

\title{
Synthesis of (+)-Haperforin G
}

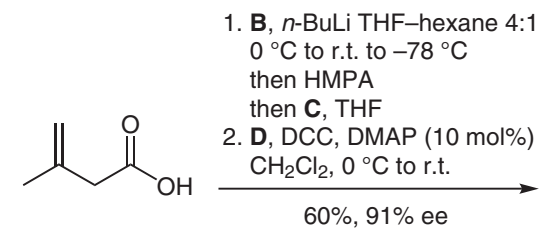

A
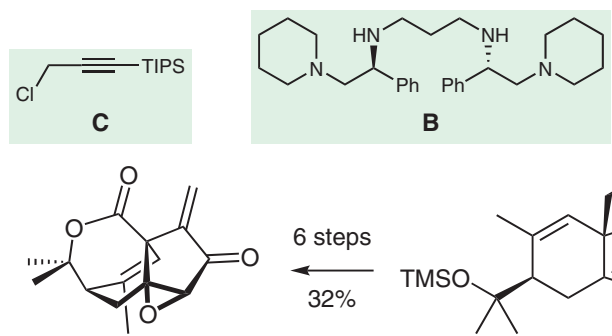

I

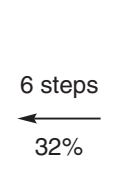

TMSO

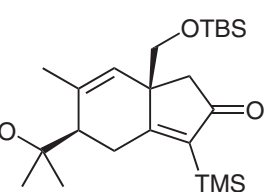

H<smiles>C=C[C@H](C[O])OC(=O)[C@H](CC#CC(F)F)C(=C)C</smiles>

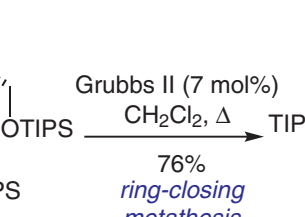<smiles>CC1=C[C@H](C[OH2+])OC(=O)C1CC#CS</smiles>
metathesis

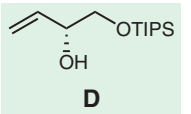

$\mathrm{Co}_{2}(\mathrm{CO})_{8}(20 \mathrm{~mol} \%)$ $\mathrm{PhMe}$ then $\mathrm{CO}(1 \mathrm{~atm}), 120^{\circ} \mathrm{C}$ $86 \%$

Pauson-Khand reaction

1. $\mathrm{Pd}(\mathrm{OAc})_{2}(10 \mathrm{~mol} \%)$ DPPF (30 mol\%)

$\mathrm{PhMe}, 130^{\circ} \mathrm{C}$

2. Jones reagent

acetone $-\mathrm{H}_{2} \mathrm{O}(2.3: 1)$

$0{ }^{\circ} \mathrm{C}$ to r.t.

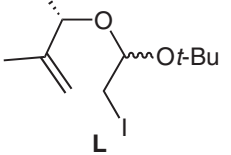

L

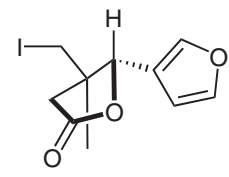

M

\section{Category}

Synthesis of Natural

Products and

Potential Drugs

\section{Key words}

(+)-haperforin G

limonoids

tetranortriterpenoids

ring-closing

metathesis

Pauson-Khand reaction

light-mediated radical coupling

aldol addition

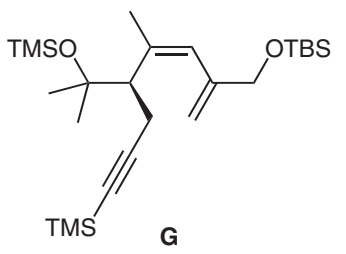

Synfact

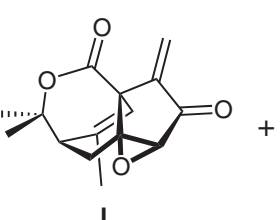

K

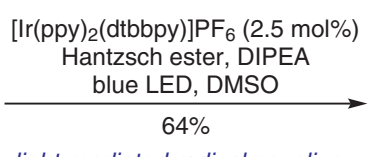

light-mediated radical coupling

M

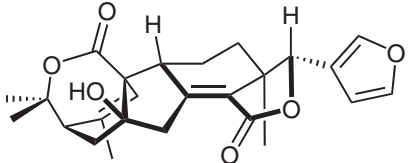

(+)-Haperforin G

1. pyridine- $\mathrm{SOCl}_{2}(10: 1), 0^{\circ} \mathrm{C}$ 2. DBU, THF, $50^{\circ} \mathrm{C}$ $50 \%$

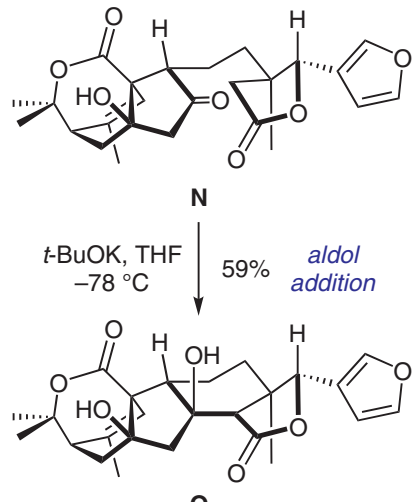

0
Significance: Chen, Yang, and co-workers report the first total synthesis of (+)-haperforin G, a structurally complex member of the limonoid family of tetranortriterpenoid natural products. Their synthesis features an efficient radical coupling of two fragments.
Comment: Intermediate $\mathbf{G}$ is accessed from $\mathbf{A}$ in nine steps and transformed to $\mathbf{H}$ via a PausonKhand reaction. Further six steps give rise to I, which is then joined with $\mathbf{M}$ via a light-mediated radical-based coupling to obtain $\mathbf{0}$. 